\title{
INFORME BIBLIOGRÁFICO: NIETZSCHE Y LA EDUCACIÓN
}

\author{
Bibliographic Report: Nietzsche and Education
}

Acampora, Christa Davis, «'Thus spoke Zarathustra' as postmodern Bildungsroman» en Nietzsche, Postmoderne und danach? eds. Endre Kiss, Uschi NussbaumerBenz Cuxhaven: Dartford, 2000, 33-41.

Allen, Ansgar. "Awaiting education: Friedrich Nietzsche on the future of our educational institutions». Philosophical Inquiry in Education, 24(2) (2017): 197-210.

Allen, Valerie \& Axiotis, Ares: «Nietzsche on the future of education». Telos: Critical Theory of the Contemporary 11 (1998), 107-121.

Allison, David. B. Reading the new Nietzsche. Lanham: Rowman \& Littlefield, 2001.

Arendt, Dieter Der Bildungs-Philister als Übermensch : oder: 'Philister über dir, Simson!'. 1996.

Arp, Wilhelm. Nietzsches Menschenideal in unse rem Erziehungsethos. Hamburg: Ohne Verlag, 1939.

Assmann, Aleida. Arbeit am nationalen Gedächtnis: eine kurze Geschichte der deutschen Bildungsidee. Frankfurt: Campus Verlag, 1993.

Aviram, Aharon. «Nietzsche as Educator?». Journal of Philosophy of Education, 25 (2), (1991): 219-234.

Baader, Meike Sophia. Erziehung als Erlösung : Transformationen des Religiösen in der Reformpädagogik. Weinheim: Juventa Verlag ein Imprint der Julius Beltz $\mathrm{GmbH} \&$ Co. KG, 2004.

Babich, Babette. «Become the one you are: On Commandments and praise - among friends». En T. Hart (Ed.), Nietzsche, culture, and education (pp. 13-38). London: Ashgate, 2009.

Babich, Babette. «Early continental philosophy of science». In: K. Ansell-Pearson \& A. Schrift (eds), The history of continental philosophy. Volume Three, The New Century: Bergsonism, Phenomenology, and Responses to Modern Science (pp. 263-286). Durham: Acumen, 2010b. 
Barthel, Ernst. «Nietzsches LehrevomRing derZeitundihregegenwärtigeFortbildung». 1936.

Bauer-Milliken, Karin. «What does not kill me makes me stronger: Nietzsche’s and Adorno's critiques of Bildung». Germanistik Internationales Referatenorgan mit bibliographischen Hinweisen seit 1960.

Bell, Michael. «Nietzsche as Educator and the Implosion of Bildung». In Open secrets: Literature, education, and authority from J-J. Rousseau to J. M. Coetzee. Oxford: Oxford University Press, 2007.

Bertin, Giovanni Maria, Nietzsche e l'idea di educazione. Torino: Il segnalibro, 1995.

Bimberg, Siegfried «Zwischen Apokalypse und Bildungseuphorie - mit Nietzsche, Schönberg und Thomas Mann» en Bildung - Kunst und Kontemplation ed. Siegfried Ortrud Bimberg, 164-170.Frankfurt: Peter Lang GmbH, Internationaler Verlag der Wissenschaften, 2002.

Blass, Josef Leonhard. «Kritik und Neuentwurf der Bildung in Nietzsches Basler Vorträgen "Über die Zukunft unserer Bildungsanstalten"». Saeculum 28 (1977): 101-125.

Bock, Wolfgang. "Zwischen Feldzeichen und Fotografie: Zu Nietzsches Metaphorik in der Schrift über die Zukunft der Bildungsanstalten» en Hegel und Nietzsche - eine literarisch-philosophische Begegnung. Eds. Klaws Vieweg y Richard T. Gray. 94-111. Weimar: Bauhaus-Universitätsverlag Weimar, 2007.

Bohley, Reiner. «Nietzsches christliche Erziehung» Nietzsche-Studien 16 (1) (2010): 164-196.

Bohley, Reiner. «Nietzsches christliche Erziehung». Nietzsche-Studien 16 (1) (2010): 164-196.

Broene, Johannes Nietzsche's educational ideas and ideals. Dass. - Dt. Zusammenfassung u.d.T.: Nietzsches Erziehungsideen und Erziehungsideale / von George J. Lenz. 1911, 17449.

Bruford, Walter Horace. The German Tradition of Self-Cultivation: 'Bildung' from Humboldt to Thomas Mann. Cambridge: Cambridge University Press, 1975.

Büssgen, Antje. Glaubensverlust und Kunstautonomie: Über die ästhetische Erziehung des Menschen bei Friedrich Schiller und Gottfried Benn (Beiträge zur neueren Literaturgeschichte. Heidelberg: Universitätsverlag Winter GmbH Heidelberg, 2006.

Caygill, Howard. «Philosophy and cultural reform» In: K. Ansell-Pearson, \& H. Caygill, (eds), The fate of the new Nietzsche (pp. 109-122). Avebury: Ashgate, 1993.

Clemens, Manuel. «Lauschen als Bildungserlebnis in Nietzsches Vorträgen "Über die Zukunft unserer Bildungsanstalten"». Monatshefte 109 (1) (2017): 25-44.

Clemens, Manuel. Das Labyrinth der ästhetischen Einsamkeit: eine kleine Theorie der Bildung. Würzburg: Königshausen \& Neumann, 2016.

Conant, James. «Nietzsche's perfectionism: A reading of Schopenhauer as Educator» In Richard Schacht, Nietzsche's Postmoralism: Essays on Nietzsche's Prelude to Philosophy's Future (pp. 181-257). Cambridge: Cambridge University Press, 2001. 
Cooper, David. Authenticity and learning: Nietzsche's educational philosophy. London: Routledge, 1983.

Cooper, Ian. «Nietzsche, money and 'Bildung'». En Nietzsche, Power and Politics, eds. Herman W. Siemens y Vasti Roodt, 605-628. New York: Walter de Gruyter, 2008.

Cysarz, Herbert. Von Schiller zu Nietzsche: Hauptfragen der Dichtungs- und Bildungsgeschichte des jüngsten Jahrhunderts. Halle (Saale): Max Niemeyer, 1928

Dammer, Karl-Heinz. «Friedrich Nietzsche - An den Grenzen von Erziehung und Bildung». Philosophen als pädagogische Denker. Ed. Barbara Budrich, 146189. Leverkusen: $\quad$ Budrich, 2017.

Dammer, Karl-Heinz. «Friedrich Nietzsche - An den Grenzen von Erziehung und Bildung». En Philosophen als pädagogische Denker. Ed. Barbara Budrich, 146-189. Leverkusen: BUDRICH, 2017.

Decuble, Gabriel H. «Und es immer schlimmer machen...». Nietzsches philosophischer Entbildungsroman. Reihe GGR-Beiträge zur Germanistik, 27. Bucuresti: Editura Universităţii din București und Editura Paideia, 2012.

Dellinger, Jakob. «... auch nur ein Glaube, eine Einbildung, eine Dummheit? FW 354 zwischen, Philosophie“ und ,Literatur». En Nietzsche zwischen Philosophie und Literatur. Eds. Katharina Grätz y Sebastian Kaufmann, 255-322. Heidelberg: Universitätsverlag Winter GmbH, 2016.

Dorschel, Andreas. «Das Programm ästhetischer Erziehung bei Schiller und beim frühen Nietzsche». Vierteljahrsschrift Für Wissenschaftliche Pädagogik 68 (3):260-284 (1992).

Duke, George. «Review of Thomas Hart, ed., Nietzsche, Culture, and Education». Surrey: Ashgate, 2008. Educational Philosophy and Theory, 42(8) (2010), 918920.

Düsign, Edith. «Die Kritik des Bildungsphilisters in Nietzsches früher Philosophie: Nietzsches Auseinandersetzung mit David Friedrich Strauß». Humanität und Bildung (1988): 186-202.

Düsing, Edith. «Wer wird das Bild des Menschen aufrichten“"?!. Nietzsches Konzept der Selbstbildung im Dialog mit Sokrates, Pascal und Schiller». En Perspektiven der Philosophie: Neues Jahrbuch. Band 39, ed. Geroges Goedert und Martina Scherbel, 81-106. Amsterdam: Rodopi, 2013.

Düsing, Edith. Die Problematik des Ichbegriffs in der Grundlegung der Bildungstheorie : Aspekte der Konstitution von personaler Identität bei Dilthey, Nietzsche und Hegel. Verlag: Köln, 1977.

Fairfield, Paul. «Nietzsche and Self-Education». In M. Peters (Ed.), Encyclopedia of Educational Philosophy and Theory. New York: Springer, 2017.

Figl, Johann. «Die "Ausbildung der Seele erkennen" : Die Bedeutung der frühen Texte Nietzsches innerhalb seiner Philosophie im ganzen». En Nietzscheforschung, Jahrbuch der Nietzsche-Gesellschaft, 5/6 (2000): 433-442.

Figl, Johann. Nietzsche und die Religionen: transkulturelle Perspektiven seines Bildungs- und Denkweges. Berlín: Walter de Gruyter, 2007. 
Finke-Lecaudey, Geneviève. «Poetische Verfahren der Wortbildung in "Also sprach Zarathustra"». En Cahiers d'Etudes Germaniques 23 (1992): 47-59.

Fitzsimons, Peter. «Nietzsche's Ubermensch as a Metaphor or Education». Paideusis, 16(1) (2007): 5-15.

Gamm, Hans-Jochen. «Nietzsches Kritik der Bildung im Horizont der Gegenwart und deren Perspektive auf Europa». En Nietzsche und die Kultur - ein Beitrag zu Europa? Gefälligkeitsübersetzung: Nietzsche and culture - a contribution towards Europe?, eds. Georges Goedert y Uschi Nussbaumer-Benz, 67-89. Hidersheim: Olms, 2002.

Gamm, Hans-Jochen. «Nietzsches Kritik der Bildung im Horizont der Gegenwart und deren Perspektive auf Europa». En Nietzsche und die Kultur - ein Beitrag zu Europa? / hrsg. von Georges Goedert und Uschi Nussbaumer-Benz. Hildesheim; Zürich, 2002, pp. 67-89.

Ganzoni, Wener. Die neue Schau der Seele: Goethe, Nietzsche, Klages : mit 2 Abbildungen und Schriftproben. Wien; Stuttgart: Braumüller, 1957.

Gellhaus, Axel. «"Ästhetische Erziehung des Menschengeschlechts": Lessing und Schiller bei Nietzsche» en Klassik, modern eds. Georg Guntermann, Jutta Osinski y Hartmut Steinecke, 112-121. Berlin: Schmidt, 1996.

Gennari, Mario. Storia della Bildung: formazione dell'uomo e storia della cultura in Germania e nella Mitteleuropa. Brescia: La Scuola, 1995.

Goehler, Josef. «Nietzsche: seine Bedeutung für die Leibeserziehung.». N-S Sport, 30 (2) (1942).

Goldberg, Richard. «Nietzsche und Tolstoi als Schaffende am Erziehungsproblem». Monatshefte für deutsche Sprache und Pädagogik 13 (3) (1912): 69-74.

Golik-Szarawarska, Grażyna. «Wagnerianismus in den Übersetzungen der Griechischen Dramatischen Dichtung von Tadeusz Zieliński». International Journal of Musicology, 1 (2015): 15-46.

Gordon, Haim. «Nietzsche's Zarathustra as Educator» Journal of Philosophy of Education, 14(2) (1980), 181-192.

Gordon, Moderchai. «Teachers as Absurd Heroes: Camus' Sisyphus and the Promise of Rebellion». Educational Philosophy and Theory, 48(6) (2016), 589-604.

Gramm, Hans-Jochen. «Friedrich Nietzsche und Walter Flez: Bildung im nationalistichen Gefälle. En Nietzsche in der Pädagogik? Beiträge zur Rezeption und Interpretation, eds. Christian Niemeyer et al., 120.131. Weinheim: Detscher Studien Verlag, 1998.

Gray, John. «Anti-Education by Friedrich Nietzsche». The Guardian, 8 January (2016).

Grummes. Ulrich. Das Problem der Bildung : eine Auseinander-setzung mit dem Denken des jungen Nietzsche. München: Ludwig-Maximilians-Universität zu München, 1971.

Günzel, Stephan. «Naturgeschichte als Vorbild und Herausforderung. Grundlagen historiographischer Modellbildung bei Kant und Nietzsche». En Kant und Nietzsche im Widerstreit, ed. Beatrix Himmelmann, 260-276. Berlín: De Gruyter, 2005. 
Gutschidt, Holger. «'Bildungsanstalten’ beim frühen Nietzsche Die Universitätsidee Nietzsches zwischen Fichte und Humboldt». En BAND 12 Bildung - Humanitas Zukunft bei Nietzsche (Nietzscheforschung), ed. Renate Reschke, 97-110. Berlín: De Gruyter, 2005.

Gutschmidt, Holger. "Von der "Idee einer Universität" zur "Zukunft unserer Bildungsanstalten" - Bildungsbegriff und Universitätsgedanke in der deutschen Phi-losophie von Schiller bis Nietzsche». En Bildung als Kunst, eds. Jürgen Stozenberg y Lars-Thade Ulrichs, 149-168. Berlín: De Gruyter, 2010.

Haase, Ullrich Michael. «Über die Zukunft unserer Bildungsanstalten in bedürftiger Zeit.». En BAND 7 Nietzscheforschung Band 7 (Nietzscheforschung), ed. Renate Reschke, 71-88. Berlín: De Gruyter, 2000.

Hadot, Pierre. Philosophy as a way of life. Oxford: Blackwell, 1995.

Hart, Thomas. (ed.). Nietzsche, culture, and education. Surrey: Ashgate, 2008.

Havenstein, Martin. «Nietzsche als Erzieher». In M. Oehler (Ed.), Den Manen Friedrich Nietzsches (pp. 91-108). Munchen: Musarion, 1921.

Heilke, Thomas: Nietzsche's tragic regime: culture, aesthetics, and political education. Illionis: Northern Illinois University Press, 1998.

Heinrich, Caroline. «Über die gegenwärtige Lage unserer Bildungsanstalten». En Sonderband 2 Nietzsche - Radikalaufklärer oder radikaler Gegenaufklärer? (Nietzscheforschung), ed. Renate Reschke, 343-350. Berlín: De Gruyter, 2004.

Heintel, Erich. «Der Raum der Erziehung bei Nietzsche» en Erkenntnis und Erzihung eds. Ernst Klett y J.G. Cotta, 29-43. Stuttgart: Klett-Cotta, 1978.

Henckmann, Eva. Grundlagen der Bildungsphilosophie im Frühwerk Nietzsches. Berlín: Verlag Münster, 2014.

Hetzel, Christoph. Der Leib als Subjekt der Bildung: Nietzsches und Merleau-Pontys Anthropologien in bildungstheoretischer Sicht. Berlín: Wissenschaftlicher Verlag, 2011.

Himmelmann, Beatrix. «Selbststeigerung. Nietzsches Idee der Bildung». En Bildung als Kunst, eds. Jürgen Stolzenberg and Lars-Thade Ulrichs, 191-212. Berlín: De Gruyter, 2010.

Hödl, Hans Gerald. «Musik, Wissenschaft und Poesie im Bildungsprogramm des jungen Nietzsche oder: Man ist über sich selbst entweder mit Scham oder mit Eitelkeit ehrlich». En Nietzsche und die Musik, eds. Günther Pöltner y Helmuth Vetter. Bristol: Peter Lang, 1997.

Hödl, Hans Gerald. «Vom kleinen Stockphilister zum Kritiker der greisenhaften Jugend "Reflexionen zum Kontext von Bildungsprogramm und Selbstentwürfen Nietzsches 1858-1865 Selbst ein Entwurf». Nietzscheforschung 5-6, no. JG (1998); 369-382.

Hotter, Karl. Das Bildungsproblem in der Philosophie Nietzsches. Inaugural Dissertation, München, 1958.

Hoyer, Timo. «"Höherbildung des ganzen Leibes”: Friedrich Nietzsches Vorstellungen zur Körpererziehung.» Nietzsche-Studien 32 (2003): 59-77.

Hoyer, Timo. Nietzsche und die Padagogik: Werk, Biografie und Rezeption. Wurzburg: Konigshausen \& Neumann, 2002. 
Hufnagel, Erwin. « Nietzsche als Provokation für die Bildungsphilosophie: Versuch, den "Griechischen Staat" zu lessen». En BAND 7 Nietzscheforschung Band 7 (Nietzscheforschung), ed. Renate Reschke, 37-58. Berlín: De Gruyter, 2000.

Janaway, Christopher. (Ed.) Willing and nothingness: Schopenhauer as Nietzsche's Educator. Oxford: Oxford University Press, 1999.

Jannsen, Horst. Hundert Köpfe. Herausgegeben von Dierk Lemcke. München: Deutscher Taschenbuch Verlag, 1994.

Johansson, Viktor., \& Schumann, Claudia. «Bildung, self-cultivation, and the challenge of democracy: Ralph Waldo Emerson as a Philosopher of Education». Educational Philosophy and Theory, 49 (2017): 1-4.

Jöhnck, Johannes. Ressentiment - Eine erziehungswissenschaftliche Studie im Anschluss an Friedrich Nietzsche und Theodor W. Adorno. Frankfurt: Johann W. Goethe Universität - Dekanat, 2015.

Johnston, James. Scott. «Nietzsche as educator: A reexamination». Educational Theory, 48(1) (2005): 67-83.

Jonas, Mark. E. "Advancing equality and individual excellence: The case of Nietzsche's "Schopenhauer as Educator"». History of Philosophy Quarterly, 33(2) (2016): 173-192.

Jonkoff, Thodor. Nietzsches Idee vom Übermenschen als Erziehungsideal. Diss. Zürich 1911.

Klass, Tobias, \& Kokemohr, R. "“Man muß noch Chaos in sich haben, um einen tanzenden Stern gebären zu können": Bildungstheoretische Reflexionen im Anschluß an Nietzsches "Also sprach Zarathustra. Ein Buch für Alle und Keinen”». En Nietzsche in der Pädagogik, ed. Ch. Niemeyer et al., 280-324. Weinheim: DSV, 1998.

Kluge, Hans. Die Bildungsidee in den Schriften des jungen Nietzsche. Frankfurt: Johan Wolfgang Goethe Universität, 1955.

Kohlmeyer, Otto, Nietzsche und das Erziehungsproblem Versuche einer einfachen systematischen Fassung der wichtigsten Gedanken Friedrich Nietzsches über Erziehung. Frankfurt: Diesterweg, 1925.

Kokemohr, Rainer. Zukunft als Bildungsproblem : die Bildungsre-flexion des jungen Nietzsche. Düsseldorf: A. Henn Verlag Ratingen, 1973.

Kolk, Rainer. Literarische Gruppenbildung. Am Beispiel des George-Kreises 1890 1945. Berlín: Max Niemeyer Verlag, 1998.

Kühn, Lenore. «Nietzsches Willenserziehung». Der Panther 5 (1917): 519-535.

Kühn, Rolf. «Einbildung als Imago mundi bei Nietzsche». En Wie das Leben spricht: Narrativität als radikale Lebensphänomenologie, 159-196. Switzerland: Springer International Publishing, 2002.

Lange, Georg. Erziehung als Zeugung. Zum Aufbau deutscher Kultur. Berlín: München, Verl. Ernst Reinhardt, 1928.

Larrosa, Jorge. «Cómo se llega a ser lo que se es: Nietzsche y el estallido de la idea de "Bildung"», en Instantes y Azares, 1(2001), 15-38.

Lemco, Gary. Nietzsche as Educator. Lanham: Edward Mellen Press, 1992.

Liessmann, Konrad Paul. «Bildungskurven: von Humboldt über Hegel zu Nietzsche und Adorno». En Formen der Bildung. Einblicke und Perspektiven. 
Gefälligkeitsübersetzung: Forms of education. Insights and perspectives, ed. Martina Schmidhuber, 39-52. Frankfurt, Main: P. Lang, 2010.

Lindemann, H. «Unsere Kultur und Jugendbildung im Lichte Nietzsches». En Pädagogis Studien, 45 (1924): 96-106, 148-162.

Lohwasser, Diana. «Das Dasein als ästhetisches Phänomen: Ästhetische Bildung als kritisch-reflexive Lebenspraxis bei Friedrich Nietzsche». En Geschichte der ästhetischen Bildung. Eds. Jörg Zirfas, Leopold Klepacki y Diana Lohwasser, 257-275. Paderborn: Verlag Ferdinand Schöningh, 2016.

Löw, Reinhard, Nietzsche - Sophist und Erzieher : philosophische Untersuchungen zum systematischen Ort von Friedrich Nietzsches Denken. Weinheim: Acta humaniora, 1984. - XIII, 222.

Löw, Reinhard, Selbsterziehung und Übermensch : Reflexionen über Friedrich Nietzsches Pädagogik. 1993.

Löw, Reinhard. Nietzsche als Sophist und Erzieher. Philosophische Untersuchungen zum systematischen Ort von F. Nietzsches Denken. Weinheim: Acta Humanioria, 1984.

Löwisch, Dieter-Jürgen. «Der freie Geist: Nietzsches Umwertung der Bildung für die Suche nach einer zeit-gemäßen Bildungsvorstellung.». En Nietzsche in der Pädagogik? Beiträge zur Rezeption und Interpretation, 1998.

Mahr, Herbert. «Einige Aspekte der kritischen Analyse von Bildungsund Erziehungsauffassungen Friedrich Nietzsches» en Beiträge zur Kritikder bürgerlichen Phiilosophie und Gesellschaftstheorie, eds. Rolf Bauermann y Dieter Bergner, 165-172. Halle: Martin-Luther-Universität Halle - Wittenberg, 1987.

Mahrhold, Werner. Friedrich Nietzsche und die Idee der Hochschule als einer Bildungsanstalt. Ars Bd. 4 (1916): 449-457.

Mannsfeldt, E. «Friedrich Nietzsche und die Spracherziehung der deutschen Jugend: ein Beitrag zur Erneuerung des Aufsatzunterrichts». En Die MittelShule 51 (1937): 135-138, 148-151.

Marquardt, Katrin. Zur sozialen Logik literarischer Produktion : die Bildungskritik im Frühwerk von Thomas Mann, Heinrich Mann und Hermann Hesse als Kampf um symbolische Macht. Würzburg: Königshausen \& Neumann, 1997.

Mattioli, William. «Das Unbewusste als transzendentaler Raum perspektivischer Weltbildung bei Nietzsche». En Nietzsches Philosophie des Unbewussten, eds. Jutta Georg y Clauss Zittel, 173-184. Berlín De Gruyter, 2012.

Meyer, Friedrich. «Die aktuelle Bedeutung der Gedanken Nietzsches über Kulturund Bildungsform im Schlußabschnitt seiner "Zweiten Unzeitgemäßen"». En Nationalsozialistiches Bildungswesen, 5, 8. München, 1940.

Meyer, Herman. Der Bildungsphilister. Berlín: De Gruyter, 1948.

Meyer, Richard. «Nietzsches Wortbildungen». En Zeitschrift für deutsche Wortforschung Band 15 (1914): 98-146.

Murphy, Timothy F., Nietzsche as educator, London: University Press of America, 1984.

Naake, Erhard. «Die Beziehungen zwischen Elisabeth Förster-Nietzsche und dem Thüringischen Innen- und Volksbildungsminister Wilhelm Frick.». En Weimar 
1930: Politik und Kultur im Vordfeld der NS-Diktatur, eds. Von Ehrlich Lothar y John Jürgen, 275-292. Köln: Weimar; Wien, 1998.

Nicolai, Heinz. Die Bildungsidee des jungen Nietzsche. M. Doesterweg, 1937.

Niemeyer, Christian. "“Erziehung ist Umtaufen-lernen oder Anders-fühlen lernen":

Nietzsches pädagogische Erfahrung als Hintergrund seiner Muttersuche.» en Nietzsche auf der Couch ed. Niemeyer, Christian 92-105. Weinhein: Beltz Verlag, 2017.

Niemeyer, Christian. "NNichts ist wahr, alles ist erlaubt”: Die Wahrheitstheorie Nietzsches in ihrer Bedeutung für seine späte Bildungsphilosophie». En Band 27 (1998): 196-213.

Niemeyer, Christian. «Jenseits des Übermenschen:Nietzsches späte Psychologisierung eines bildungsphilos-ophischen Konstrukts». En Vierteljahrsschrift für wissenschaftliche Pädagogik 71, 4 (1999): 396-411.

Niemeyer, Christian. «Nietzsches "Bildungsvorträge" von 1872: einige Deutungshinweise zu einem übe-raus fragwürdigen Text». En BAND 12 Bildung - Humanitas - Zukunft bei Nietzsche, ed. Renate Reschke, 35-52. Berlín: De Gruyter, 2005.

Niemeyer, Christian. «Nietzsches Vortäge "ÜberdieZukunftunsererBildungsanstalten” im Kontext: kritische An-merkungen aus rezeptionsgeschichtlicher Perspektive mit Schwerpunkt auf Wagner, La-garde und Langbehn». En Vierteljahrsschrift für wissenschaftliche Pädagogik, 75 (1999): 173-207.

Niemeyer, Christian. Et al (eds)., Nietzsche in der Pädagogik? : Beiträge zur Rezeption und Interpretation. Weinheim: やDeutscher Studien Verlag, 1998.

Niemeyer, Christian. Wie wurde mit Nietzsche im 20. Jahrhundert Bildungspolitik gemacht?: Ein Rückblick auf gut einhundert Jahre Rezeptionsgeschichte. En BAND 12 Bildung - Humanitas - Zukunft bei Nietzsche, ed. Renate Reschke, 1330. Berlín: De Gruyter, 2005.

Niemeyer, Christian: «"Erziehung ist Umtaufen-lernen oder Anders-fühlen lernen”: Nietzsches pädagogische Erfahrung als Hintergrund seiner Muttersuche», en Neue Sammlung. Vierteljahres-Zeitschrift für Erziehung und Gesellschaft. Jg. 36. Seelze-Velber 1996. H. 2, S. 223-243.

Niemeyer, Christian: "Nichts ist wahr, alles ist erlaubt" : Die Wahrheitstheorie Nietzsches in ihrer Bedeutung für seine späte Bildungsphilosophie», en Nietzsche-Studien, 2011.

Nietzsche, Friedrich. «Homer and classical philology». New Nietzsche Studies, 10(3 \& 4) (2017): 113-125.

Nietzsche, Friedrich. In C. Wellmon \& P. Reitter (eds), Anti-Education. New York: New York Review of Books, 2015.

O'Hara, D. T. The prophet of our laughter: Or Nietzsche as educator?. Boundary 2, Vol. 9/10: 1-21. Vol. 9, no. (3) Vol. 10, no. 1 (1981), Why Nietzsche Now? A Boundary 2 Symposium (Spring-Autumn).

Oelkers, Jürgen. «Einige Bemerkungen Friedrich Nietzsches über Erziehung und der Status eines "Klassikers der Pädagogik".» en Nietzsche in der Pädagogik? eds. Herausgegeben von Christian Niemeyer, Heiner Drerup, Jürgen Oelkers y Lorenz v. Pogrell, 211-240. Weinheim: Deutscher Studien Verlag, 1998. 
Owen, David. «Equality, democracy, and self-respect: Reflections on Nietzsche's Agonal Perfectionism». The Journal of Nietzsche Studies, 24/(1) (2002): 113131.

Patoussis, Stavros. "Winckelmann in Nietzsches Vorträgen "Ueber die Zukunft unserer Bildungsanstalten": zur Figuration des 'Bildungsführers' und seiner Selbstaufhebung». Nietzscheforschung 24 (1) (2017): 123-144.

Peters, Michael, Marshall, James, \& Smeyers, Paul. (eds). Nietzsche's legacy for education: Past and present values. Westport, CT: Bergin \& Garvey, 2001.

Poenitsch, Andreas. Bildung und Sprache zwischen Moderne und Postmoderne: Humboldt, Nietzsche, Ballauff, Lyotard. Berlín: Verlag Die Blaue Eule, 1992.

Ränsch-Trill, Barbara. Phantasie: Welterkenntnis und Welterschaffung - zur philosophischen Theorie der Einbildungskraft. Bouvier: Bonn, 1996.

Rasch, William. «Wissenschaftsphilister: Nietzsche über das moderne Bildungswesen». En Philister Problemgeschichte einer Sozialfigur der neueren deutschen Literatur, eds. Remigius Bunia, Till Dembeck and Georg Stanitzek, 383-396. Berlín: De Gruyter, 2011.

Rattner, J. Nietzsche als Erzieher, Nietzsche-Forschung. Berlin: Akademie Verlag, 1994.

Rese, Friederike. «Horizontbildung und Weltbildung: zur Mensch-Tier-Differenz in Heideggers "Grundbegriffe der Metaphysik"-Vorlesung». En Heidegger \& Nietzsche, eds. Babette Babich, Alfred Denker, and Holger Zaborowski, 391415. Boston: Brill, 2012.

Rodríguez, Mariano L., «Elementos para una pedagogía nietzscheana», en Revista de filosofia, 9 (1986): 37-48 y 257-268.

Rosenow, Eliyahu. "'Nietzsche als Erzieher' kontra 'Nietzsche in der Padagogik?'». Ein Vergleich der anglo-amerikanischen und der deutschen NietzscheInterpretationen am Vorabend des 21. Jahrhunderts. Zeitschrift Fur Padagogik, 46(6) (2000): 867-879.

Rosenow, Eliyahu: «Nietzsche's concept of education». En Nietzsche as affirmative thinker: papers presented at the Fifth Jerusalem Philosophical Encounter, April 1983 / ed. by Yirmiyahu Yovel, Boston: Dordrecht, 1986: 119-131.

Rott, Joseph. "Nietzsche und die klassische Bildung». En Blätter für deutsche Erzichung, 14 (1912): 85-88, 202-211.

Rowthorn, David. «Nietzsche's Cultural Elitism». Canadian Journal of Philosophy, 47(1) (2017): 97-115.

Salaquarda, Jörg. «Man ist nicht umsonst Philologe gewesen ...”: zur Bildungskritik Friedrich Nietzsches». Berichte zur WissenschaftsgeschichteVolume 11, 3 (1988): 179-189.

Salzsieder, Paul. «Die Auffassungen und Weiterbildungen der Schopenhauerschen Philosophie». En Annalen der Philosophie Und Philosophischen Kritik 7 (1928):148-149.

Sauerland, Karol. «Der Bildungsgedanke des jungen Nietzsche». En Nietzscheforschung, 7, (2000): 31-36.

Schacht, Richard: "Zarathustra/"Zarathustra" as educator», en Nietzsche: a critical reader / ed. by Peter R. Sedgwick. Oxford: Cambridge, 1995, pp. 222-249. 
Schäfer, Alfred. «Genealogie - Macht - Bildung». En BAND 7 Nietzscheforschung Band 7 (Nietzscheforschung), ed. Renate Reschke, 59-70. Berlín: De Gruyter, 2000 .

Schmid, Wolfgang. Die Grundlegung der Bildung im Denken des jungen Friedrich Nietzsche. Köln, 1971.

Schmidt-Millard, Torsten. Nietzsches Basler Vorträge "Über die Zukunft unserer Bildungsanstalten": die Aporie der Bildungstheorie des "Genius" und ihre Über-windung in den "Unzeitge-mäßen Betrachtungen”. Köln: Universität zu Köln, 1982.

Schnädelbach, Herbert. «Nietzsches Kritik der historischen Bildung». En Études germaniques: revue trimestrielle de la Société des Études Germaniques, 55, 2 (2000): 169-184.

Schneider, Jörg. Nietzsches Basler Vorträge "Über die Zukunft unserer Bildungsanstalten” im Lichte seiner Lektüre pädagogischer Schriften. Köln: Universität Zu Köln, 1982.

Stein, Alfred. «Die Stellung des jungen Nietzsche zu den Fragen der Erziehung und Bildung». En Pädagogisches Archiv 52 (1910): 73-99.

Stoeckert, Hedwig. Nietzsche und das Problem der Erziehung. Langensalza: Hermann

Strong, Tracy. B. Friedrich Nietzsche and the politics of transfiguration. Berkeley: University of California Press, 2000.

Thiele, Leslie. P. «The educator and the solitary». En: Friedrich Nietzsche and the politics of the soul: A study of heroic individualism (pp. 165-182). Princeton: Princeton University Press, 1990.

Thirring, Hans. Anti Nietzsche - Anti Spengler. Gesammelte Aufsätze und Reden zur demokratischen Erziehung. Viena: Sexl Verlag, 1947.

Voigt, Günther. «Nietzsches Gedanken über Bildung und Erziehung». En Neue Jahrbücher für deut- sche Wissenschaft 13 (1937): 558-568.

Wallaschek, Richard. «Nietzsche über Erziehung». En Monatsschirft für christliche Sozialreform 33 (1911): 302-303.

Weeks, Marcus. What Would Nietzsche Do? London: Hachette, 2017.

Wessely, Rudolf. «Friedrich Nietzsche als Vorkämpfer für Erziehung und Schulreform». Pädagogisches Archiv 54 (1912): 601-631.

Winterer, Konrad. «Friedrich Nietzsche und die Bestrebungen der Blätter für deutsche Erziehung». - In: Blätter für deutsche Erziehung 3 (1910).

Winterfeld, Achim von. Unser Erziehungswesen in der Auffassung Nietzsche's. Leizipg: Gautzch b., 1910.

Cristina Alvarado Díaz

Universidad de Málaga 